\title{
Structure of PEP-PEO block copolymer micelles: Exploiting the complementarity of small-angle X-ray scattering and static light scattering
}

\author{
Jensen, Grethe Vestergaard; Shi, Qing; Hernansanz, Maria J.; Oliveira, Cristiano L. P.; Deen, G. Roshan; \\ Almdal, Kristoffer; Pedersen, Jan Skov
}

Published in:

Journal of Applied Crystallography

Link to article, DOI:

10.1107/S0021889811013343

Publication date:

2011

Document Version

Publisher's PDF, also known as Version of record

Link back to DTU Orbit

Citation $(A P A)$ :

Jensen, G. V., Shi, Q., Hernansanz, M. J., Oliveira, C. L. P., Deen, G. R., Almdal, K., \& Pedersen, J. S. (2011). Structure of PEP-PEO block copolymer micelles: Exploiting the complementarity of small-angle X-ray scattering and static light scattering. Journal of Applied Crystallography, 44(3), 473-482.

https://doi.org/10.1107/S0021889811013343

\section{General rights}

Copyright and moral rights for the publications made accessible in the public portal are retained by the authors and/or other copyright owners and it is a condition of accessing publications that users recognise and abide by the legal requirements associated with these rights.

- Users may download and print one copy of any publication from the public portal for the purpose of private study or research.

- You may not further distribute the material or use it for any profit-making activity or commercial gain

- You may freely distribute the URL identifying the publication in the public portal 
Journal of

\section{Applied Crystallography}

ISSN 0021-8898

Received 4 September 2010

Accepted 9 April 2011

\section{Structure of PEP-PEO block copolymer micelles: exploiting the complementarity of small-angle X-ray scattering and static light scattering}

\author{
Grethe Vestergaard Jensen, ${ }^{a}$ Qing Shi, ${ }^{a}$ María J. Hernansanz, ${ }^{a}$ Cristiano L. P. \\ Oliveira, ${ }^{\mathrm{a}} \neq$ G. Roshan Deen, ${ }^{\mathrm{a}} \mid$ Kristoffer Almdal $^{\mathrm{b}}$ and Jan Skov Pedersen ${ }^{\mathrm{a} *}$

\begin{abstract}
a Department of Chemistry and Interdisciplinary Nanoscience Centre (iNANO), Aarhus University, Langelandsgade 140, DK-8000 Aarhus C, Denmark, and ${ }^{\mathbf{b}}$ DTU Nanotech, Department of Microand Nanotechnology, Technical University of Denmark, Building 345Ø, DK-2800 Kongens Lyngby, Denmark. Correspondence e-mail: jsp@chem.au.dk
\end{abstract}

\begin{abstract}
The structure of large block copolymer micelles is traditionally determined by small-angle neutron scattering (SANS), covering a large range of scattering vectors and employing contrast variation to determine the overall micelle morphology as well as the internal structure on shorter length scales. The present work shows that the same information can be obtained by combining static light scattering (SLS) and small-angle X-ray scattering (SAXS), which provide information on, respectively, large and short length scales. Micelles of a series of block copolymers of poly(ethylene propylene)- $b$-poly(ethylene oxide) (PEP-PEO) in a $70 \%$ ethanol solution are investigated. The polymers have identical PEP blocks of $5.0 \mathrm{kDa}$ and varying PEO blocks of $2.8-49 \mathrm{kDa}$. The SLS contrasts of PEP and PEO are similar, providing a homogeneous contrast, making SLS ideal for determining the overall micelle morphology. The SAXS contrasts of the two components are very different, allowing for resolution of the internal micelle structure. A core-shell model with a PEP core and PEO corona is fitted simultaneously to the SAXS and SLS data using the different contrasts of the two blocks for each technique. With increasing PEO molecular weight, a transition from cylindrical to spherical micelles is observed. This transition cannot be identified from the SAXS data alone, but only from the SLS data.
\end{abstract}

(C) 2011 International Union of Crystallography Printed in Singapore - all rights reserved
2005). The balance between forces that results in the selfassembled structures is very delicate and thus depends strongly on the actual conditions. A wide variety of micellar and bilayer shapes and sizes are observed depending on, for example, solvent, temperature, salt concentration and pH (Cammas et al., 1997; Hanley et al., 2000; Lee et al., 2002).

Small-angle scattering techniques are essential for studying micelle structures. For small-angle X-ray scattering (SAXS) the origin of the contrast that gives rise to the scattering is the difference in electron density between the particles and the surrounding medium. The contrast can be different for the micelle core and corona, which leads to a high structural information content in the scattering pattern.

The typical values of scattering vector lengths $q(q=$ $4 \pi \sin \theta / \lambda$, where $2 \theta$ is the scattering angle and $\lambda$ is the X-ray wavelength) covered are from 0.003 to $0.35 \AA^{-1}$ depending on $\mathrm{X}$-ray wavelength, detector size and detector-sample distance. According to the Fourier transformation sampling theorem, information is lost for sizes larger than about $\pi / q_{\min }$ (Shannon \& Weaver, 1959; Bracewell, 2000; Moore, 1980; Glatter \& Kratky, 1982). For SAXS this means that the maximum size we can determine is $\sim 1000 \AA$. For block copolymer micelles,
† Present address: Instituto de Física, Universidade de São Paulo, Caixa Postal 66318, 05314-970 São Paulo, Brazil.

- Present address: Natural Science and Science Education, National Institute of Education, Nanyang Technological University, 1-Nanyang Walk, Singapore. 
however, the micellar sizes can easily exceed this size for polymers of high molecular weight and for micelles that do not have a simple spherical shape. This means that important information on the overall shape and size might not be obtained from SAXS. One possible way to retrieve this additional information is to combine SAXS with static light scattering (SLS) measurements, which in our case cover the $q$ region $0.00035-0.0026 \AA^{-1}$. SLS thus provides scattering from almost one decade of $q$ in the region just below the region accessible by SAXS and reaches up to a micrometre in size. Therefore, additional information on large micelles can be obtained by combining SLS and SAXS. Furthermore, the SLS contrast is given by excess polarizability; this can be very different from the contrast for SAXS, which is given by the excess electron densities.

Here a combination of SAXS and SLS is applied for the determination of micelle structures for a series of block copolymers of poly(ethylene propylene)-block-poly(ethylene oxide) (PEP-PEO).

PEP-PEO block copolymer micelles in aqueous solution have previously been studied in great detail by small-angle neutron scattering (SANS) (Poppe et al., 1997). The effect of increasing the PEO block length has been investigated, and spherical micelles with an increasingly star-like structure were identified (Willner et al., 2000). Upon increasing the total molecular weight of the block copolymer it has been shown that the shape of the micelles goes from being cylindrical to spherical (Kaya et al., 2002). Also the effect of decreasing the surface tension of the solvent towards the core by adding dimethylformamide has been studied and shown to give smaller aggregation numbers of micelles (Lund et al., 2004; Stellbrink et al., 2004; Laurati et al., 2007).

In the present study the solvent is a water/ethanol mixture of $70 \%$ ethanol, and we investigate the effect of changing the PEO molecular weight from 2.8 to $49 \mathrm{kDa}$ while keeping the PEP molecular weight constant at $5.0 \mathrm{kDa}$. By combining SAXS and SLS we obtain scattering information for different contrast conditions without changing the degree of deuteration of the solvent as is necessary when performing contrast variation with SANS. We give the basic equations for the scattering contrasts of SLS and SAXS, and it is explained how the contrasts are determined experimentally. The expressions for an advanced core-shell model with internal structure of the shell (corona) are given, and the application of the model to the scattering data is described. The SLS and SAXS data were fitted simultaneously by the same structural model in which the difference in scattering contrast is taken into account. The derived results are presented and discussed in terms of free energy considerations for the micelle structures. The series of block copolymers displays a transition from a cylindrical to a spherical shape as a function molecular weight of the PEO block due to the increasing importance of the PEO inter-chain interactions in the corona for increasing size of the PEO blocks. The work demonstrates the advantages of exploiting the complementarity of the SLS and SAXS techniques and the associated different $q$ ranges and contrast conditions.

\section{Contrasts for SLS and SAXS}

When calculating the scattering cross section for a core-shell model for the micelles, the excess scattering length density for, respectively, the chains in the core and in the corona enters the expressions. For SAXS the excess scattering length density is proportional to the electron density difference relative to that of the solvent. In general one can write the excess scattering length density of species $i, \Delta \rho_{\mathrm{SAXS}, i}$, as

$$
\Delta \rho_{\mathrm{SAXS}, i}=b_{\mathrm{e}}\left(\rho_{\mathrm{e}, i}-\rho_{\mathrm{e}, \text { solvent }}\right),
$$

where $b_{\mathrm{e}}$ is the electron scattering length $\left(2.82 \times 10^{-13} \mathrm{~cm}\right.$, the classical Thomson radius), $\rho_{\mathrm{e}, i}$ is the electron density (electrons per unit volume) of species $i$ and $\rho_{\mathrm{e}, \text { solvent }}$ is the electron density of the solvent. The electron densities of the solvent and of the two blocks can be obtained from measurements of solvent and solution densities (Sommer et al., 2004). The apparent partial specific density of a polymer in solution, $\rho_{\text {polymer }}$, can be found from the density of the solution, $\rho_{\text {solution }}$, and the density of the pure solvent, $\rho_{\text {solvent }}$, as

$$
\rho_{\text {solution }}=\frac{m_{\text {polymer }}+m_{\text {solvent }}}{m_{\text {polymer }} / \rho_{\text {polymer }}+m_{\text {solvent }} / \rho_{\text {solvent }}},
$$

where $m_{\text {polymer }}$ and $m_{\text {solvent }}$ are the masses of polymer and solvent in the solution. Note that the apparent partial specific density of the polymer is an effective density that takes into account effects of changes of the solvent density in the vicinity of the blocks under the assumption that the solvent has bulk density.

When apparent densities for both a PEP-PEO block copolymer and a homopolymer of PEO are determined, the contributions from PEP and PEO to the apparent density of PEP-PEO can be separated (Sommer et al., 2004). It is assumed that the apparent density of PEO in the micelle is the same as for the PEO homopolymer. Any deviation from this will influence the determined apparent density of PEP, $\rho_{\text {PEP. }}$. The equation relating the apparent densities of PEP-PEO, PEO and PEP is then

$$
\rho_{\mathrm{PEP}-\mathrm{PEO}}=\frac{m_{\mathrm{PEP}}+m_{\mathrm{PEO}}}{m_{\mathrm{PEP}} / \rho_{\mathrm{PEP}}+m_{\mathrm{PEO}} / \rho_{\mathrm{PEO}}},
$$

where $m_{\mathrm{PEP}}$ and $m_{\mathrm{PEO}}$ are the masses of PEP and PEO in the block copolymer.

The electron densities can be calculated from the apparent densities for each of the polymer blocks:

$$
\begin{aligned}
& \rho_{\mathrm{e}, \mathrm{PEP}}=\rho_{\mathrm{PEP}} N_{\mathrm{A}} Z_{\mathrm{PEP}} / M_{\mathrm{PEP}}, \\
& \rho_{\mathrm{e}, \mathrm{PEO}}=\rho_{\mathrm{PEO}} N_{\mathrm{A}} Z_{\mathrm{PEO}} / M_{\mathrm{PEO}},
\end{aligned}
$$

and similarly for the solvent

$$
\begin{aligned}
\rho_{\mathrm{e}, \text { solvent }}= & \rho_{\text {solvent }} N_{\mathrm{A}}\left[\varphi_{\mathrm{ethanol}} Z_{\text {ethanol }} / M_{\text {ethanol }}\right. \\
& \left.+\left(1-\varphi_{\text {ethanol }}\right) Z_{\text {water }} / M_{\text {water }}\right]
\end{aligned}
$$

where $Z_{i}$ and $M_{i}$ denote, respectively, the number of electrons and the molecular weight of species $i$, and $N_{\mathrm{A}}$ is Avogadro's number. $\varphi_{\text {ethanol }}$ is the weight fraction of ethanol in the solvent. As mentioned above, the measured specific densities are apparent values that include effects of solvent density changes 
that might be associated with the polymer dissolution. Effects such as selective sorption of one of the solvent components in the corona are not taken into account in equations (4) and (5), and the electrons of PEP, PEO and the solvent have been allocated to the apparent specific volumes $1 / \rho$ of the respective species.

For SLS the excess scattering length density is related to the refractive indices of the components and it can be written as (Pusey, 2002)

$$
\begin{aligned}
\Delta \rho_{\mathrm{SLS}, i} & =\frac{k^{2}}{4 \pi}\left(\frac{n_{i}^{2}-n_{\text {solvent }}^{2}}{n_{\mathrm{s}}^{2}}\right) \simeq \frac{k^{2}}{2 \pi n_{\mathrm{s}}}\left(n_{i}-n_{\text {solvent }}\right) \\
& =\frac{2 \pi n_{\mathrm{s}}}{\lambda_{0}^{2}}\left(n_{i}-n_{\text {solvent }}\right)=\frac{2 \pi n_{\mathrm{s}}}{\lambda_{0}^{2}} \frac{\mathrm{d} n_{\mathrm{s}}}{\mathrm{d} c_{i}} \rho_{i},
\end{aligned}
$$

where $n_{i}, n_{\text {solvent }}$ and $n_{\mathrm{s}}$ are, respectively, the refractive indices of species $i$, of the solvent and of the solution. $k=2 \pi / \lambda$ is the length of the wavevector, where $\lambda$ is the light wavelength in the solution, which is related to the light wavelength in vacuum $\lambda_{0}$ by the solution refractive index $n_{\mathrm{s}}: \lambda=\lambda_{0} / n_{\mathrm{s}} \mathrm{d} n_{\mathrm{s}} / \mathrm{d} c_{i}$ is the refractive index increment per concentration unit of the species $i$, and $\rho_{i}$ is the density of the species $i$. The final expression is valid under the assumption that the refractive index of the solution is a volume-weighted average of the refractive indices of its constituents. This gives $n_{\mathrm{s}}=\sum_{i}\left(\varphi_{i} n_{i}\right)+$ $\left(1-\sum_{i} \varphi_{i}\right) n_{\text {solvent }}$ and $\mathrm{d} n_{\mathrm{s}} / \mathrm{d} c_{i}=\left(\mathrm{d} n_{\mathrm{s}} / \mathrm{d} \varphi_{i}\right)\left(\mathrm{d} \varphi_{i} / \mathrm{d} c_{i}\right)=\left(n_{i}-\right.$ $\left.n_{\text {solvent }}\right) / \rho_{i}$, where $\varphi_{i}$ is the volume fraction of species $i$ in the solution. That is, $n_{i}-n_{\text {solvent }}=\left(\mathrm{d} n_{\mathrm{s}} / \mathrm{d} c_{i}\right) \rho_{i}$, which is used in equation (6).

The equations show that the contrast can be calculated on the basis of either refractive indices or refractive index increments. The refractive index of a polymer in solution is difficult to obtain experimentally and might deviate substantially from that of the same polymer in bulk owing to perturbation by the solvent in the vicinity of the polymers. When using refractive index increments, the contrasts for polymers in solution are obtained, which gives more reliable values. Values of $\mathrm{d} n_{\mathrm{s}} / \mathrm{d} c_{i}$ for both PEP and PEO in the solvent have to be determined. Since PEP is not soluble in the solvent, the refractive index increment for this block cannot be measured directly. If we again consider the refractive index of the solution, $n_{\mathrm{s}}$, as a volume-weighted average of the refractive indices of its constituents we obtain

$$
n_{\mathrm{s}}=\varphi_{\mathrm{PEO}} n_{\mathrm{PEO}}+\varphi_{\mathrm{PEP}} n_{\mathrm{PEP}}+\left(1-\varphi_{\mathrm{PEO}}-\varphi_{\mathrm{PEP}}\right) n_{\mathrm{solvent}},
$$

where $\varphi_{\mathrm{PEP}}$ and $\varphi_{\mathrm{PEO}}$ are the volume fractions in the solution of PEP and PEO, respectively. $n_{\mathrm{PEP}}$ and $n_{\mathrm{PEO}}$ are the corresponding apparent refractive indices. This gives the apparent refractive index increments (as explained above)

$$
\frac{\mathrm{d} n_{\mathrm{s}}}{\mathrm{d} c_{\mathrm{PEO}}}=\frac{\left(n_{\mathrm{PEO}}-n_{\text {solvent }}\right)}{\rho_{\mathrm{PEO}}} \quad \text { and } \quad \frac{\mathrm{d} n_{\mathrm{s}}}{\mathrm{d} c_{\mathrm{PEP}}}=\frac{\left(n_{\mathrm{PEP}}-n_{\text {solvent }}\right)}{\rho_{\mathrm{PEP}}} .
$$

Letting $\varphi$ be the total volume fraction of polymer and $x$ the volume fraction of PEO in the polymer, the solution refractive index can also be written as

$$
n_{\mathrm{s}}=\varphi\left[x n_{\mathrm{PEO}}+(1-x) n_{\mathrm{PEP}}\right]+(1-\varphi) n_{\text {solvent }},
$$

giving an apparent refractive index increment for PEP-PEO of

$$
\begin{aligned}
\frac{\mathrm{d} n_{\mathrm{s}}}{\mathrm{d} c_{\text {polymer }}} & =\frac{\mathrm{d} n_{\mathrm{s}}}{\mathrm{d} \varphi} \frac{1}{\rho_{\text {polymer }}} \\
& =\frac{1}{\rho_{\text {polymer }}}\left[x\left(n_{\mathrm{PEO}}-n_{\text {solvent }}\right)+(1-x)\left(n_{\mathrm{PEP}}-n_{\text {solvent }}\right)\right] \\
& =\frac{1}{\rho_{\text {polymer }}}\left[x \frac{\mathrm{d} n_{\mathrm{s}}}{\mathrm{d} c_{\mathrm{PEO}}} \rho_{\mathrm{PEO}}+(1-x) \frac{\mathrm{d} n_{\mathrm{s}}}{\mathrm{d} c_{\mathrm{PEP}}} \rho_{\mathrm{PEP}}\right]
\end{aligned}
$$

This shows that $\mathrm{d} n_{\mathrm{s}} / \mathrm{d} c_{\text {PEP }}$ can be deduced from the refractive index increment of PEO and of PEP-PEO. The assumption employed is that $\mathrm{d} n_{\mathrm{s}} / \mathrm{d} c_{\text {PEO }}$ for PEO in the micelle is equal to the value for the homopolymer PEO in the same solvent. Note that a similar assumption was made about the apparent partial specific densities when deriving the SAXS contrasts.

Note that the measured and calculated refractive index increments of PEO and PEP are apparent values in the sense that they include any effects that might arise from changes in the solvent in the vicinity of the polymers. The possibility of selective sorption is not included in the calculation of the contrasts, which considers only PEP, PEO and a homogeneously mixed solvent.

\section{Models}

As the micelles occur with both spherical and cylindrical shapes, models for these two shapes have to be considered. Both models describe core-corona particles with a dense core and a swollen corona. Molecular constraints are used as far as possible in the models in order to reduce the number of fit parameters. From the knowledge of the apparent partial specific densities and the molecular weights of the blocks, effective volumes of the two blocks can be calculated for a given block copolymer. For a given aggregation number $p$ of the micelles, the total effective volumes of the core blocks $V_{\text {core }}$ and the corona blocks $V_{\mathrm{PEO}, \text { tot }}$ are given by, respectively, $V_{\text {core }}=p V_{\mathrm{PEP}}$ and $V_{\mathrm{PEO} \text {,tot }}=p V_{\mathrm{PEO}}$, where $V_{\mathrm{PEP}}$ and $V_{\mathrm{PEO}}$ are the volumes of the PEP and PEO blocks, respectively. Since the solvent is strongly selective for PEO and a non-solvent for PEP, it can furthermore be assumed that the core volume is equal to that of the PEP blocks $V_{\text {core }}$. For spherical micelles, the radius of the core $R_{\text {core }}$ was used as a fit parameter. This gives $V_{\text {core }}=4 \pi R_{\text {core }}^{3} / 3$ and the aggregation number $p=V_{\text {core }} /$


fit parameters and $V_{\text {core }}=\pi R_{\text {core }}^{2} L$ with the same expression for the aggregation number $p=V_{\text {core }} / V_{\text {PEP. }}$

For the spherical micelles, the normalized scattering amplitude $\left[A_{\mathrm{sph}}(q=0)=1\right]$ for the core is (Rayleigh, 1910)

$$
A_{\text {sph }}\left(q R_{\text {core }}\right)=\frac{3\left[\sin \left(q R_{\text {core }}\right)-q R_{\text {core }} \cos \left(q R_{\text {core }}\right)\right]}{\left(q R_{\text {core }}\right)^{3}} .
$$

The normalized scattering amplitude for a spherical shell representing the micelle corona of inner radius $R_{\text {core }}$ and outer radius $R_{\text {mic }}$ is given as 


$$
A_{\text {sph shell }}\left(q, R_{\text {core }}, R_{\text {mic }}\right)=\frac{A_{\text {sph }}\left(q R_{\text {mic }}\right) R_{\text {mic }}^{3}-A_{\text {sph }}\left(q R_{\text {core }}\right) R_{\text {core }}^{3}}{R_{\text {mic }}^{3}-R_{\text {core }}^{3}} .
$$

To obtain the total scattering form factor for the spherical micelles (Pedersen \& Gerstenberg, 1996; Svaneborg \& Pedersen, 2002), the amplitudes from the core and the corona are weighted by the respective PEP and PEO volumes $V_{i}$ and contrasts $\Delta \rho_{i}$, summed and then squared:

$$
\begin{aligned}
& P_{\text {sph mic }}(q)=\left[p V_{\mathrm{PEP}} \Delta \rho_{\mathrm{PEP}} A_{\mathrm{sph}}\left(q R_{\mathrm{core}}\right)\right]^{2} \\
& +p\left[p-P_{\text {chain }}^{\prime}(0)\right]\left[V_{\mathrm{PEO}} \Delta \rho_{\mathrm{PEO}} A_{\text {sph shell }}\left(q, R_{\text {core }}, R_{\mathrm{mic}}\right)\right]^{2} \\
& +2 p^{2} V_{\mathrm{PEP}} \Delta \rho_{\mathrm{PEP}} A_{\mathrm{sph}}\left(q R_{\mathrm{core}}\right) V_{\mathrm{PEO}} \Delta \rho_{\mathrm{PEO}} A_{\text {sph shell }}\left(q, R_{\mathrm{core}}, R_{\mathrm{mic}}\right) \\
& +p\left(V_{\mathrm{PEO}} \Delta \rho_{\mathrm{PEO}}\right)^{2} P_{\text {chain }}^{\prime}(q) .
\end{aligned}
$$

In this expression $P_{\text {chain }}^{\prime}(q)$ is the effective scattering form factor of a chain in the corona (see below). It is added to account for the characteristic chain scattering of the swollen corona. The weight of the second term, stemming from the corona shell, is reduced corresponding to the forward chain scattering $P_{\text {chain }}^{\prime}(0)$ to ensure the correct total PEO scattering.

For the cylindrical micelles, the scattering form factor of the core is given by that of a cylinder, $P_{\text {cyl }}\left(q, R_{\text {core }}, L\right)$. For $L>>$ $R_{\text {core }}$, the scattering from the cylinder length and cross section can be decoupled (Porod, 1948; Pedersen \& Schurtenberger, 1996a) into a product

$$
P_{\text {cyl }}\left(q, R_{\text {core }}, L\right)=\left|A_{\text {cs }}\left(q R_{\text {core }}\right)\right|^{2} P_{\text {length }}(q L),
$$

with

$$
A_{\text {cs }}\left(q R_{\text {core }}\right)=2 J_{1}\left(q R_{\text {core }}\right) /\left(q R_{\text {core }}\right)
$$

and

$$
P_{\text {length }}(q L)=2 \operatorname{Si}(q L) /(q L)-4[\sin (q L / 2) /(q L)]^{2},
$$

where $J_{1}(x)$ is a first-order Bessel function of the first kind and $\operatorname{Si}(x)=\int_{0}^{x} t^{-1} \sin t \mathrm{~d} t$ (Neugebauer, 1943). The form factor of the corona is also written as decoupled contributions from the cross-sectional and longitudinal scattering, which is valid when $L>R_{\text {mic }}$ :

$P_{\text {cyl shell }}\left(q, R_{\text {core }}, R_{\text {mic }}, L\right)=\left|A_{\text {cs shell }}\left(q, R_{\text {core }}, R_{\text {mic }}\right)\right|^{2} P_{\text {length }}(q L)$,

where

$$
A_{\mathrm{cs} \mathrm{shell}}\left(q, R_{\mathrm{core}}, R_{\mathrm{mic}}\right)=\frac{A_{\mathrm{cs}}\left(q R_{\mathrm{mic}}\right) R_{\mathrm{mic}}^{2}-A_{\mathrm{cs}}\left(q R_{\mathrm{core}}\right) R_{\mathrm{core}}^{2}}{R_{\mathrm{mic}}^{2}-R_{\mathrm{core}}^{2}} .
$$

$A_{\text {cs shell }}\left(q, R_{\text {core }}, R_{\text {mic }}\right)$ is the scattering amplitude of the corona shell of the cylinder cross section, and end effects have been neglected. Note that the expressions (11), (12) and (14)-(18) are normalized to unity at $q=0$.

For the cylindrical micelles the amplitudes from the crosssectional scattering are summed and squared, and then multiplied by $P_{\text {length }}$ (see also Pedersen, 2000), which gives

$$
\begin{aligned}
& P_{\text {cyl mic }}(q)=P_{\text {length }}(q L)\left\{\left[p V_{\mathrm{PEP}} \Delta \rho_{\mathrm{PEP}} A_{\mathrm{cs}}\left(q R_{\text {core }}\right)\right]^{2}\right. \\
& +p\left[p-P_{\text {chain }}^{\prime}(0)\right]\left[V_{\mathrm{PEO}} \Delta \rho_{\mathrm{PEO}} A_{\text {cs shell }}\left(q, R_{\text {core }}, R_{\mathrm{mic}}\right)\right]^{2} \\
& \left.+2 p^{2} V_{\mathrm{PEP}} \Delta \rho_{\mathrm{PEP}} A_{\mathrm{cs}}\left(q R_{\text {core }}\right) V_{\mathrm{PEO}} \Delta \rho_{\mathrm{PEO}} A_{\text {cs shell }}\left(q, R_{\text {core }}, R_{\text {mic }}\right)\right\} \\
& +p\left(V_{\mathrm{PEO}} \Delta \rho_{\mathrm{PEO}}\right)^{2} P_{\text {chain }}^{\prime}(q)
\end{aligned}
$$

As in equation (13), $P_{\text {chain }}^{\prime}(q)$ describes the scattering of a chain in the corona, and the weight of the second term from the corona shell is reduced corresponding to $P_{\text {chain }}^{\prime}(0)$ to ensure the correct total PEO scattering.

The expressions given above for the form factors assume sharp interfaces between core and corona and between corona and solvent, which is unrealistic. Both the core and the corona surfaces are expected to be smeared, and this is incorporated into the model by, for the spherical micelles, multiplying the scattering amplitudes $A_{\text {sph }}\left(q R_{\text {core }}\right)$ and $A_{\text {sph }}\left(q R_{\text {mic }}\right)$ with the Gaussian functions $\exp \left(-\sigma_{\text {core }}^{2} q^{2} / 2\right)$ and $\exp \left(-\sigma_{\text {corona }}^{2} q^{2} / 2\right)$, respectively. The corona surface is highly smeared; however, $\sigma_{\text {corona }}$ is limited to be below one-third of the corona thickness to avoid negative corona contributions close to the corecorona interface, which would invalidate the volume conservation in the model. For the cylindrical micelles, it is $A_{\text {cs }}\left(q R_{\text {core }}\right)$ and $A_{\mathrm{cs}}\left(q R_{\text {mic }}\right)$ that are multiplied by $\exp \left(-\sigma_{\text {core }}^{2} q^{2} / 2\right)$ and $\exp \left(-\sigma_{\text {corona }}^{2} q^{2} / 2\right)$, respectively.

An attempt to use a linear combination of three cubic $b$ spline functions for describing the radial profile of the corona (Pedersen et al., 2003) was also made. However, this increased the number of fit parameters and the quality of the fits did not improve compared to the corona profile with Gaussian smearing. In addition, the fitted radial profiles of the two approaches did not deviate substantially, and therefore only the results from fitting with the Gaussian-smeared corona are shown.

The effective single-chain form factor of the PEO chains in the corona enters the form factor expressions for both spherical and cylindrical micelles. It has been shown (Svaneborg \& Pedersen, 2001) that the function is well described by a random phase approximation (RPA) expression that takes into account the concentration effects within the corona:

$$
P_{\text {chain }}^{\prime}(q)=\frac{P_{\text {chain }}(q)}{1+v P_{\text {chain }}(q)},
$$

where $P_{\text {chain }}(q)$ is the single-chain form factor, for which we used a numerical expression based on a Monte Carlo simulation that takes both semi-flexibility and self-avoidance into account (Pedersen \& Schurtenberger, 1996b; Pedersen et al., 1996). It is a function of the contour length $L_{\mathrm{PEO}}$, which can be estimated from the chemical structure, and the Kuhn length, which was set to $10 \AA$ (Pedersen \& Gerstenberg, 1996). The interaction parameter $v$ can be estimated from the density of chains on the core surface. It is a function of the reduced surface coverage $\Sigma / \Sigma^{*}$ (Svaneborg \& Pedersen, 2001), which is the two-dimensional equivalent of the reduced concentration $c / c^{*}$ in bulk solution. The parameter $c / c^{*}$ is the concentration relative to the overlap concentration $c^{*}$ of the polymers. $\Sigma$ is the surface area coverage of the PEO chains in 
the corona of a micelle if each chain covers an area of $\pi R_{\mathrm{g}, \mathrm{PEO}}^{2}$, where $R_{\mathrm{g}, \mathrm{PEO}}$ is the radius of gyration of the random-walk PEO chain. The overlap coverage $\Sigma^{*}$ is the available area at a distance $R_{\mathrm{g}, \mathrm{PEO}}$ from the core surface. For the aggregation number $p$ of the micelles, we get the expressions for $\Sigma / \Sigma^{*}$ :

$$
\begin{aligned}
\text { Spheres: } \Sigma / \Sigma^{*} & =\frac{p R_{\mathrm{g}, \mathrm{PEO}}^{2}}{4\left(R_{\text {core }}+R_{\mathrm{g}, \mathrm{PEO}}\right)^{2}}, \\
\text { Cylinders: } \Sigma / \Sigma^{*} & =\frac{p R_{\mathrm{g}, \mathrm{PEO}}^{2}}{2\left(R_{\text {core }}+R_{\mathrm{g}, \mathrm{PEO}}\right) L} .
\end{aligned}
$$

For corona polymer chains in a good solvent it has been shown by Monte Carlo simulations that $v\left(\Sigma / \Sigma^{*}\right)=1.42\left(\Sigma / \Sigma^{*}\right)^{1.04}$ (Svaneborg \& Pedersen, 2002). In order to apply this dependence of $v$ on $\Sigma / \Sigma^{*}$, we need to relate the solvent condition of PEO in $70 \%$ ethanol to the good solvent conditions of the simulations. This was done by performing SAXS measurements on semi-dilute solutions of PEO homopolymers with molecular masses of 2.15, 5.40, 10.5, 20.4 and $40.0 \mathrm{kDa}$ in $70 \%$ ethanol and for each of the molecular weights analysing the SAXS data by the RPA expression (16). In these fits an expression for $v$ from renormalization group theory as a function of $x=M_{\mathrm{PEO}} A_{2} c_{\mathrm{PEO}}$ was used as this makes the RPA expression valid in the semi-dilute regime (Wang et al., 2002; Ohta \& Oono, 1982; Garamus et al., 2000). $A_{2}$ is the second virial coefficient of PEO in the given solvent and was a fit parameter. The fits were performed simultaneously to data sets from solutions of three different concentrations (1,2 and $5 \mathrm{wt} \%$ ). We also analysed simulation data (Pedersen \& Schurtenberger, 1999) for semi-dilute solutions of chains in a good solvent by the same expression and obtained a ratio of 0.760 between the experimentally determined interaction parameters and the values from the simulation. Hence we use $\nu\left(\Sigma / \Sigma^{*}\right)=0.760 \times 1.42\left(\Sigma / \Sigma^{*}\right)^{1.04}$ in the model expression for the micelles.

The intensity on the absolute scale of the models is

$$
I(q)=n P(q),
$$

where $n$ is the particle number density and $P(q)$ is the form factor of the micelles as given by equations (13) or (19). The particle number density is simply given as $n=c /(p M)$, where $c$ is the mass concentration and $M$ is the molecular weight of a polymer chain.

Size polydispersity was included in the models by a Gaussian distribution of core radii. For the cylindrical micelles an exponentially decaying distribution was applied for the length (Cates, 1987). This distribution was cut at a minimum value of $4 R_{\text {core }}$ and at a maximum value of $3 L$. The number density when including polydispersity is $n=c /(\langle p\rangle M)$, where $\langle p\rangle$ is the number-average aggregation number.

A hard-sphere structure factor (Kinning \& Thomas, 1984) was included in the model for spherical micelles to account for concentration effects. For the cylindrical micelles the influence of concentration is neglected in the model.

The model contains the following parameters that were calculated from other parameters or were fixed during the least-squares fitting (see also comments below): (i) The densities of PEO and solvent for the calculation of PEO volume and for SAXS and SLS contrasts [equations (1), (4) and (6)] were fixed at the measured values.

(ii) The refractive index increments for SLS contrast calculations [equation (6)] were fixed at the experimentally determined values.

(iii) The width of the Gaussian function for core-corona interface smearing, $\sigma_{\text {core }}$, was fixed at $5.0 \AA$.

(iv) The width of the Gaussian distribution of radii for polydispersity, $\sigma_{R \text { core }}$, was fixed at $0.08 R_{\text {core }}$.

(v) The PEO interaction parameter $v$ was calculated as a function of $\Sigma / \Sigma^{*}$ as described above.

Four (five for the cylindrical micelles) structural parameters were optimized in the fits:

(i) The micelle core radius $R_{\text {core }}$ (and micelle length $L$ for cylindrical micelles).

(ii) The volume fraction $\varphi_{\mathrm{PEO}}$ of PEO in the corona at the core-corona interface, which together with the core sizes also gives the corona thickness.

(iii) The PEP density for the calculation of PEP volume and for SAXS and SLS contrasts [equations (1), (4) and (6)]. It proved necessary to fit this parameter, which is reasonable since the solvation of PEP, and therefore also its apparent partial specific density, can be expected to vary with the core size.

(iv) The width of the Gaussian functions for smearing of the outer corona surface: $\sigma_{\text {corona }}$. As described above, it was given a maximum limit of one-third of the corona thickness.

For the spherical micelles the ratio $S_{\mathrm{HS}}$ between the hardsphere radius and the micelle radius $R_{\text {mic }}$ was also optimized. This gives the hard-sphere radius and allows for calculation of the hard-sphere volume fraction, which are the parameters in the hard-sphere structure factor.

Parameters giving the polymer concentrations for SAXS and SLS samples (in weight percent), $\varphi_{\text {polymer,SAXs }}$ and $\varphi_{\text {polymer,SLS }}$, were optimized to correct for small errors in the concentration and in the absolute scale of the data. For SAXS it stayed close to the expected value of 0.01 . For SLS it was considerably lower than the expected concentration (see Results) owing to the repeated filtration of this sample (see Experimental). A background for the SAXS data was also added.

The model was fitted simultaneously to the SAXS and SLS data by means of standard least-squares methods (Pedersen, 1997). For the SAXS data, the model was convoluted by the instrumental resolution function to take into account the instrumental smearing (Pedersen et al., 1990). The parameters of the resolution function were determined from the width of the beam at the detector (Pedersen, 1993).

\section{Experimental}

The block copolymers were synthesized by anionic living polymerization (Ndoni et al., 1995). Their molecular masses were determined by size-exclusion chromatography and matrix-assisted laser desorption/ionization time-of-flight mass spectrometry. The molecular weight of the PEP block was 
determined to be $5.0 \mathrm{kDa}$. The PEO molecular weights were $2.8,4.9,10.4,20.3$ and $49.1 \mathrm{kDa}$. The corresponding polydispersity indices were determined to be $1.07,1.03,1.11,1.12$ and 1.20 , respectively. The samples will be denoted $\mathrm{PEP}_{5^{-}}$ $\mathrm{PEO}_{M}$, where the subscripts denote the molecular weights of the block in $\mathrm{kDa}$.

SAXS data for solutions of all the block copolymers were collected on the prototype of the commercially available three-pinhole NanoStar camera from Bruker AXS with a twodimensional HiSTAR gas detector (Pedersen, 2004). The wavelength of the $\mathrm{Cu} K \alpha \mathrm{X}$-rays was $1.542 \AA$. The instrument was used with small pinholes and a large sample-detector distance of $108 \mathrm{~cm}$ to have a $q$ range of $0.004-0.2 \AA^{-1}$. All measurements were performed at $296 \mathrm{~K}$. The polymer concentration was $1.00 \mathrm{wt} \%$. To determine the interaction parameter $v$ for the PEO chains in the corona, data for PEO solutions in $70 \%$ ethanol of concentrations 1,2 and $5 \mathrm{wt} \%$ were collected. The PEO homopolymers were purchased from Polymer Source, Canada. They have weight-average molecular weights of $2.15,5.40,10.5,20.4$ and $40.0 \mathrm{kDa}$ and polydispersity indices of $1.04,1.08,1.05,1.07$ and 1.15 , respectively.

Density measurements of $\mathrm{PEP}_{5}-\mathrm{PEO}_{4.9}$ and of a PEO homopolymer $\left(M_{\mathrm{PEO}}=4.60 \mathrm{kDa}\right.$, polydispersity index of 1.3 , Aldrich) in solution were performed to obtain the contrasts for the data modelling. The measurements were performed using an Anton Paar digital vibrating glass tube densitometer (DMA 5000).

In addition to the modelling described in detail in the previous section, the indirect Fourier transformation (IFT) method (Glatter, 1977; Pedersen et al., 1994) was used for obtaining model-independent information on the micelles. The IFT method provides the pair distance distribution function $p(r)$, which is a histogram of all distances between point pairs within the particles weighted by the excess electron density (which can be both positive and negative) at the points. The function goes to zero at $r=D_{\max }$, where $D_{\max }$ is the maximum distance within the scattering objects.

SLS was performed at a commercially available ALV/CGS$8 \mathrm{~F}$ goniometer (ALV GmbH, Germany) equipped with an ALV-6010/EPP multi-tau digital correlator. The instrument is equipped with an ALV-Static \& Dynamic Enhancer with fibre splitting for operation in the pseudo-cross-correlation mode. A helium-neon diode laser (JDS Uniphase) with an output power of $25 \mathrm{~mW}$ and a wavelength of $632.8 \mathrm{~nm}$ was used as the light source. The incident light was vertically polarized with respect to the scattering plane and the light intensity was regulated with a software-controlled ALV/8 steps beam attenuator. Angles from 15 to $160^{\circ}$ corresponding to a $q$ range of $0.00035-0.0026 \AA^{-1}$ were covered.

To avoid multiple scattering and detector saturation, dilution of the samples was necessary. The $1.0 \mathrm{wt} \%$ samples applied for the SAXS measurements were diluted to $0.010 \mathrm{wt} \%$. Since the SAXS samples were already dilute, it is not expected that the further dilution will change the structure of the micelles. In the dilute regime the structure is mainly determined by the interactions between the solvent and each
Table 1

Densities of PEP from model fits and corresponding SAXS and SLS contrasts calculated from equations (1)-(5) and (6)-(10), respectively.

\begin{tabular}{llll}
\hline$M_{\text {PEO }}(\mathrm{kDa})$ & $\rho_{\text {PEP }}\left(\mathrm{g} \mathrm{cm}^{-3}\right)$ & $\Delta \rho_{\text {PEP,SAXS }}\left(10^{9} \mathrm{~cm}^{-2}\right)$ & $\Delta \rho_{\text {PEP,SLS }}\left(10^{8} \mathrm{~cm}^{-2}\right)$ \\
\hline 2.8 & $0.851(2)$ & -1.07 & 2.956 \\
4.9 & $0.848(2)$ & -1.41 & 2.944 \\
10 & $0.852(7)$ & -1.00 & 2.958 \\
20 & $0.877(2)$ & 1.40 & 3.044 \\
49 & $0.90(6)$ & 3.64 & 3.125 \\
\hline
\end{tabular}

of the blocks, which are independent of the concentration. However, for the cylindrical micelles the length of the micelles may be influenced. Since the length information is contained in the SLS data, the determined value corresponds to the diluted SLS sample. The SLS samples were centrifuged and filtered several times on a $0.2 \mu \mathrm{m}$ filter, which means that the final polymer concentration is not well determined.

Refractive index increments were measured for the PEO homopolymer with a molecular weight of $4.6 \mathrm{kDa}$ and for the $\mathrm{PEP}_{5}-\mathrm{PEO}_{4.9}$ block copolymer using a WGE Dr Bures DnDc2010. The samples had concentrations of $0.1-1.0 \mathrm{mg} \mathrm{ml}^{-1}$ for the PEP-PEO solutions and $1.0-10 \mathrm{mg} \mathrm{m}^{-1}$ for the PEO solutions. The value for PEP was then found according to equation (10).

\section{Results}

A plot of the SAXS and SLS scattered intensities as a function of $q$ is shown in Fig. 1(a). The data at lower $q$ are from SLS, and the data at higher $q$ are from SAXS. The full lines are model fits performed simultaneously to the SLS and SAXS data sets for each molecular weight of PEO, $M_{\mathrm{PEO}}$. The spherical micelle model was used for all data, except for the data from the solutions of $\mathrm{PEP}_{5}-\mathrm{PEO}_{2.8}$, where this model could not fit the SLS data. Here the cylindrical micelle model was applied instead.

The solvent density was measured to be $0.8760 \mathrm{~g} \mathrm{~cm}^{-3}$. The PEO density was fixed in the fit at the value for the $4.6 \mathrm{kDa}$ PEO homopolymer in the same solvent, which was measured to be $1.168 \mathrm{~g} \mathrm{~cm}^{-3}$, giving a SAXS contrast of $\Delta \rho_{\mathrm{PEO}, \mathrm{SAXS}}=$ $2.416 \times 10^{10} \mathrm{~cm}^{-2}$. In order to obtain satisfactory fits, the apparent specific density of PEP had to be optimized. The model results show that the PEP core is large and probably quite compact for the lowest PEO molecular weight, whereas the aggregation number and the core is much smaller for higher values of $M_{\mathrm{PEO}}$. Therefore the hydration of the PEP can be expected to vary somewhat with $M_{\mathrm{PEO}}$ and it is reasonable to optimize the value of the apparent partial specific density of PEP. The optimized PEP densities are tabulated in Table 1 . The PEP density as obtained by density measurements of a $\mathrm{PEP}_{5}-\mathrm{PEO}_{4.9}$ solution and a $4.6 \mathrm{kDa} \mathrm{PEO}$ homopolymer solution [equation (3)] is $0.859 \mathrm{~g} \mathrm{~cm}^{-3}$, which is close to the fit parameter value for the $\mathrm{PEP}_{5}-\mathrm{PEO}_{4.9}$ solution. The fitted PEP densities are also quite close to a measured bulk value for a $5 \mathrm{kDa} P E P$ at $0.853 \mathrm{~g} \mathrm{~cm}^{-3}$ (by weighing $5.00 \mathrm{ml}$ ) and a published value for PEP of $0.856 \mathrm{~g} \mathrm{~cm}^{-3}$ (Fetters et al., 1994), which confirms that the fit values are credible. The SAXS contrasts calculated from the PEP and 
solvent densities are also shown in Table 1. The PEP contrasts are much smaller than the PEO contrast, and negative for the polymer micelles of lowest PEO molecular weight. For the highest values of $M_{\mathrm{PEO}}$ the apparent PEP density increases, which might be explained by the smaller core leading to increased influence of solvation effects at the surface.

The measured refractive index increments for the $4.6 \mathrm{kDa}$ PEO homopolymer and $\mathrm{PEP}_{5}-\mathrm{PEO}_{4.9}$ micelles are 0.109 and $0.135 \mathrm{ml} \mathrm{g}^{-1}$, respectively. Using equation (10) this gives an estimate of a refractive index increment for PEP of $0.161 \mathrm{ml} \mathrm{g}^{-1}$. The SLS contrast can then be calculated by using equation (6). For PEO, it is $2.73 \times 10^{8} \mathrm{~cm}^{-2}$. The values for PEP (given in Table 1) are quite close to this value, showing the homogeneous SLS contrast of the micelles.

The pair distance distribution functions obtained by IFT of only the SAXS data are shown in Fig. 1(b). The opposite signs of contrast for core and corona lead to a shoulder at around $150 \AA$ for the three lowest molecular weights of PEO. The $p(r)$ functions show that the particles are larger for increasing PEO molecular weight, as expected. For the lowest PEO molecular weight some elongation of the micelles is suggested. However, the evidence is quite weak and is observed as a long weak tail in $p(r)$ at the highest $r$. The information of the elongation is only contained at the very lowest $q$ of the SAXS data. Even here, the data points show a less pronounced trend than what is given by the cylinder model (Fig. 1a). This might be due to inter-micellar interactions that were not included in the cylinder model or shadow effects from the beam stop. As a result of the very weak evidence of elongated micelles in the SAXS data, erroneous conclusions about the micelle shape could have been drawn if these data had been used on their own.

The SLS data extend the sampled $q$ region to the low- $q$ side by almost an order of magnitude. Both core and corona have positive contrasts, meaning that partial contrast matching leading to intensity suppression at low $q$ does not take place. Therefore the overall particle shape is clearly reflected in the SLS data. The SLS data of the $\mathrm{PEP}_{5}-\mathrm{PEO}_{2.8}$ sample follow a slope of -1 in the $\log -\log I(q)$ plot (indicated by the dashed line, Fig. 1a) showing that these micelles are indeed cylindrical.

Fig. 2(a) shows the core radii $R_{\text {core }}$ and corona thicknesses $T_{\text {corona }}=R_{\text {mic }}-R_{\text {core }}$ from the model fits. The core radius decreases with $M_{\mathrm{PEO}}$ as expected since the more space accommodating PEO chain leads to a higher core curvature at the cost of a higher core surface area. The radius for the cylindrical micelles of $\mathrm{PEP}_{5}-\mathrm{PEO}_{2.8}$ is not directly comparable to the rest, since cylinders with a radius similar to a sphere radius will have a much lower core curvature and give a lower total surface area.

In several thermodynamic mean-field models of block copolymer micelles, the core and corona blocks are assumed to have end-to-end distances equal to, respectively, the core radius and corona thickness (Leibler et al., 1983; Nagarajan \& Ganesh, 1989; Balsara et al., 1991; Zhulina et al., 2005). Hence the degree of stretching or compression of the blocks is determined by comparing these sizes with the mean-square end-to-end distance of the unperturbed core and corona blocks, $r_{\text {ee-PEP }}$ and $r_{\text {ee-PEO}}$, which are also plotted in Fig. 2(a). They are calculated as $r_{\mathrm{ee}-i}=\left(L_{i} b_{i}\right)^{0.5}$, where $L_{i}$ and $b_{i}$ are the contour length and Kuhn length for PEP and PEO, respectively. For PEP a Kuhn length of $12 \AA$ is applied (Aharoni, $1983)$, whereas for PEO we used the same value $(10 \AA)$ as for the PEO chain scattering in the model (Pedersen \& Gerstenberg, 1996).

$R_{\text {core }}$ is larger than $r_{\text {ee-PEP }}$ for all micelles except for the highest value of $M_{\mathrm{PEO}}$, meaning that the PEP chains are stretched. It decreases with $M_{\mathrm{PEO}}$ and also at the transition to cylindrical micelles at low $M_{\mathrm{PEO}}$. This decrease in $R_{\text {core }}$ is

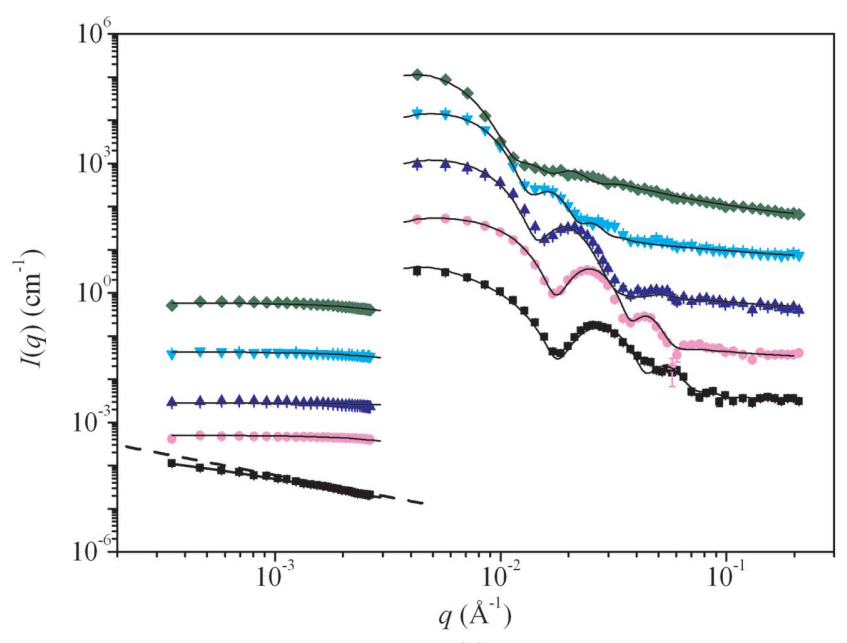

(a)

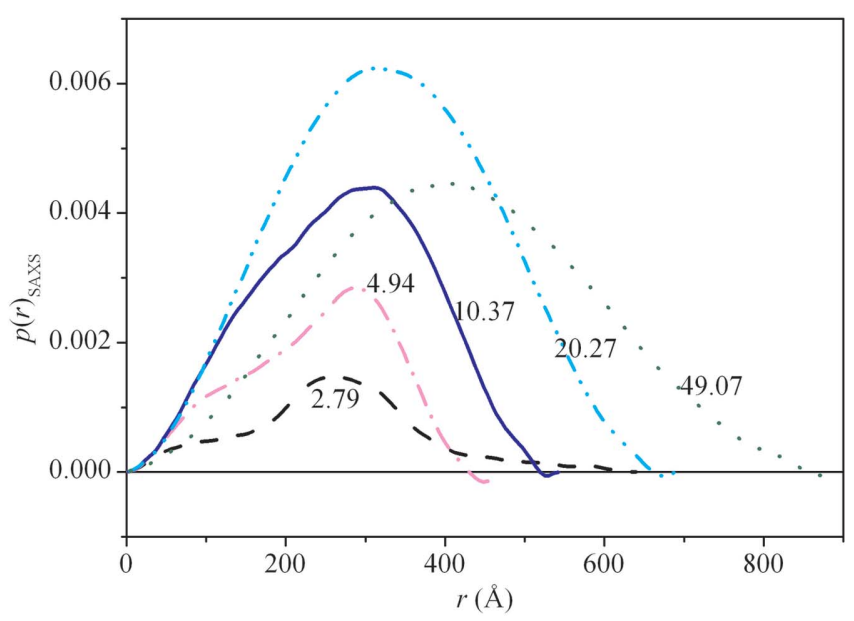

(b)

Figure 1

(a) Plot of SLS (low $q$ ) and SAXS (high $q$ ) data for PEP-PEO in $70 \%$ ethanol with $M_{\mathrm{PEP}}=5.0 \mathrm{kDa}$ and $M_{\mathrm{PEO}}$ varying from 2.8 to $49 \mathrm{kDa}$. From the bottom: $M_{\mathrm{PEO}}(\mathrm{kDa})$ : squares 2.8, circles 4.9, upward triangles 10, downward triangles 20, diamonds 49 . The data from $M_{\mathrm{PEO}}=2.8 \mathrm{kDa}$ are on an absolute scale; the rest are scaled by a factor of ten for each consecutive data set. Fits (full lines) were performed simultaneously to SLS and SAXS data for each value of $M_{\mathrm{PEO}}$. The data for $\mathrm{PEP}_{5}-\mathrm{PEO}_{2.8}$ are modelled as cylindrical micelles. The dashed line indicates the power law $I(q) \simeq q^{-1}$ expected for long cylindrical micelles. All other data sets are modelled as spherical micelles. (b) Pair distance distribution functions for the SAXS data alone of PEP-PEO in $70 \%$ ethanol with varying PEO molecular weights from 2.8 to $49 \mathrm{kDa}$ (marked on plot). 
favoured by the PEP configurational entropy, which increases when the PEP chains are less stretched.

The large corona thicknesses compared to $r_{\text {ee-PEO indicates }}$ some degree of stretching of the PEO chains due to the relatively high concentration of PEO close to the core surface, which makes it favourable for the chains to stretch to have more space as this optimizes the configurational entropy. A power-law function is fitted to the corona thickness as a function of $M_{\mathrm{PEO}}$. The exponent is determined to be 0.57 (6). This is close to the expected exponent for the mean-square end-to-end distance of the PEO chain, which is 0.588 for a selfavoiding random walk (des Cloizeaux \& Jannink, 1990).

The value of the length, $L$, of the cylindrical micelles of $\mathrm{PEP}_{5}-\mathrm{PEO}_{2.8}$ (the lowest value of $M_{\mathrm{PEO}}$ ) is $5.8(4) \times 10^{3} \AA$. The information on the length is mainly contained in the SLS data, and it should hence be interpreted as the value for the dilute solution on which SLS was performed. For this solution the modelled weight fraction of polymer was $\varphi_{\text {polymer,SLS }}=$
$0.0071(9) \mathrm{wt} \%$. The weight fraction before sample purification was $0.01 \mathrm{wt} \%$, indicating that some of the polymer was lost during the filtration and centrifugation steps.

Fig. $2(b)$ shows the volume fraction of PEO, $\Phi_{\mathrm{PEO}}$, at the core-corona interface. The results follow a power-law behaviour showing the increasing interaction between the PEO chains and the increased associated swelling with $M_{\mathrm{PEO}}$. For self-avoiding chains one can estimate the scaling of the average polymer volume fraction within a coil as $f_{\mathrm{PEO}} \simeq V_{\mathrm{PEO}} /$ $R_{\mathrm{g}, \mathrm{PEO}}^{3} \simeq M_{\mathrm{PEO}} / M_{\mathrm{PEO}}^{3 \times 0.588} \simeq M_{\mathrm{PEO}}^{-0.764}$. The exponent observed for $\Phi_{\mathrm{PEO}}\left(r=R_{\text {core }}\right)$ is -0.86 (5). The value of $\Phi_{\mathrm{PEO}}\left(r=R_{\text {core }}\right)$ for the lowest value of $M_{\mathrm{PEO}}$ deviates from the scaling behaviour, probably because of the change in morphology when the micelles go from a spherical to a cylindrical shape.

The corona profiles can be calculated as an inverse Fourier transformation of the scattering amplitude of the final fitted model (Derici et al., 1999). The profiles are plotted in Fig. 2(c) as volume fraction of PEO against distance $r$ from the micelle

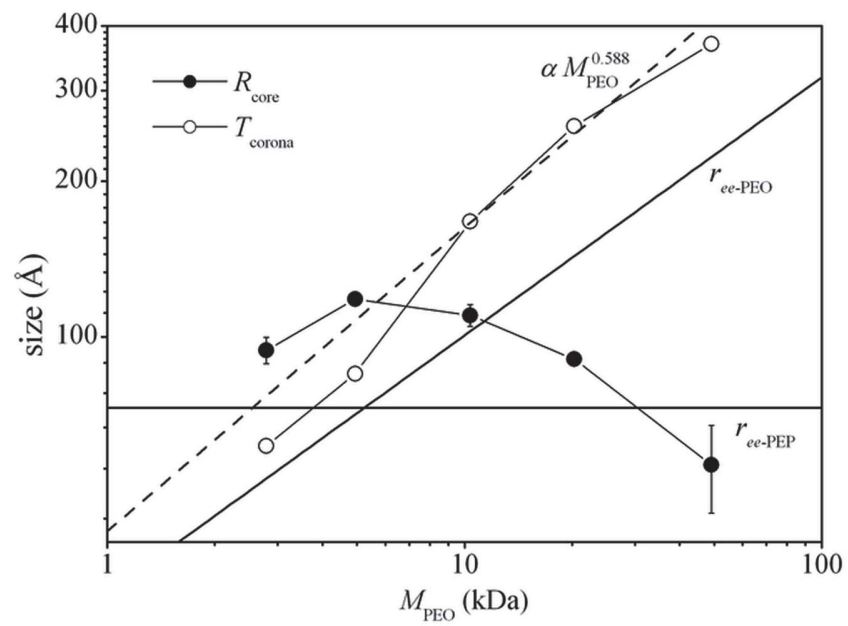

(a)

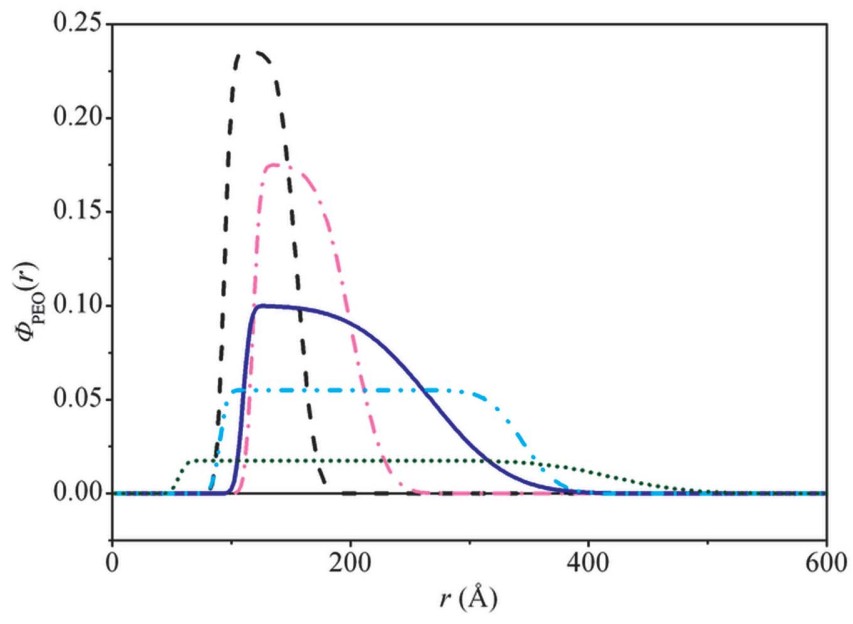

(c)

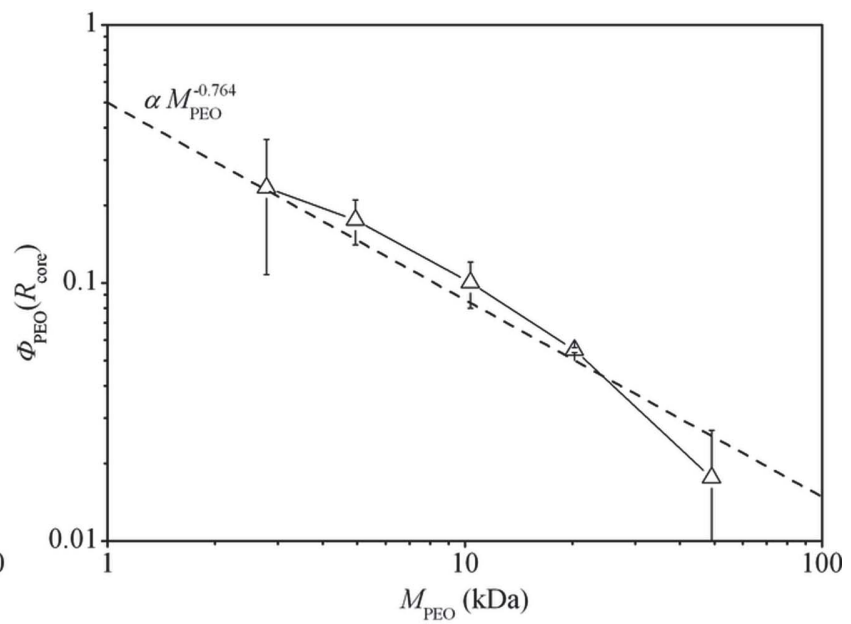

(b)

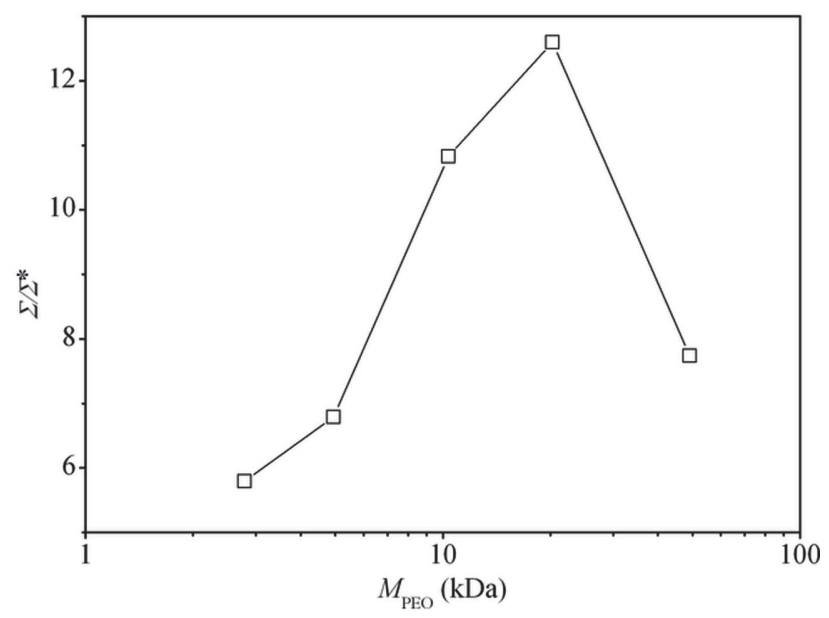

(d)

Figure 2

(a) Core radius (filled circles) and corona thickness (open circles) plotted against the molecular weight of the PEO block. The end-to-end distances expected for random-walk PEP and PEO of the given molecular weights are also shown (full lines: $r_{\text {ee-PEP }}$ and $r_{\text {ee-PEO }}$, respectively), as well as the expected power law for the PEO end-to-end distance if excluded volume is taken into account: $r_{\mathrm{ee}-\mathrm{PEO}} \simeq M_{\mathrm{PEO}}^{0.588}$ (dashed line). (b) Volume fraction of $\mathrm{PEO}$ at the core-corona interface. The dashed line indicates the expected power law for the average volume fraction of polymer within a polymer coil (see text). (c) Radial profiles of the micelle corona for different values of $M_{\mathrm{PEO}}$ : dashed 2.8, dash-dot 4.9, solid 10, dash-dot-dot 20, dotted 49 kDa. (d) Reduced surface coverage (see text). 
centre. The core profile is not shown. It has a volume fraction of unity since the PEP core is assumed not to be swollen and decays to zero where the corona starts.

The reduced surface coverage $\Sigma / \Sigma^{*}$ as calculated from equation (21) is plotted as a function of $M_{\mathrm{PEO}}$ in Fig. $2(d) . \Sigma /$ $\Sigma^{*}$ increases with $M_{\mathrm{PEO}}$ as a result of the increasing $R_{\mathrm{g}}$ of the PEO chains. At the highest $M_{\mathrm{PEO}}$, it decreases as $R_{\text {core }}$, and therefore also the aggregation number $p$, decreases. The values of $\Sigma / \Sigma^{*}$ are all much larger than unity, showing that there is strong overlap of the chains in the corona.

\section{Summary and conclusion}

In a study of PEP-PEO block copolymer micelles in a waterethanol mixture, SAXS and SLS have been combined in order to vary the contrast and obtain structural information on both overall shape and core-corona structure. For the investigated system, the contrast conditions of the micelles are very different for the two techniques. For SAXS the contrast given by the excess electron density is small for the core and in some cases even negative, whereas the corona contrast is positive. This gives a good resolution for the cross-sectional profile of the micelles. For SLS the contrast is given by the excess refractive index and is comparable and positive for core and corona. As SLS furthermore probes the low- $q$ region, this technique gives resolution of a larger length scale and thereby of the overall particle shape.

An advanced model has been fitted simultaneously to the SAXS and SLS data. The scattering contrasts of the two techniques were determined from, respectively, measurements of the apparent partial specific density and the refractive index increment. The combination of measurement on the homopolymer of the corona polymer and on micelles allowed the contrasts of the two polymer blocks to be estimated independently for both techniques.

The PEP molecular weight is constant at $5.0 \mathrm{kDa}$. For the lowest value of $M_{\mathrm{PEO}}=2.8 \mathrm{kDa}$ the micelles are cylindrical, which can only be deduced from the SLS data. For $M_{\mathrm{PEO}}$ of 4.9-49 kDa, the micelles are spherical. From the fit results it can be concluded that both the corona thickness and the corona swelling increase with $M_{\mathrm{PEO}}$. The core radius decreases to accommodate for the more spacious PEO chains. This is at the cost of increasing the core surface area. However, it is favourable for the configurational entropy of the PEP chains in the core since they become less stretched.

The combination of SAXS and SLS for contrast variation constitutes for some systems an important alternative to the traditional contrast variation performed for small-angle neutron scattering, where the contrast is varied by deuteration of one of the components of the solute and changing the degree of deuteration of the solvent. Thus, SANS requires special and expensive sample preparation and deuteration, which also has the risk that it may change the behaviour of the system (Pedersen et al., 2003). SANS contrast variation is furthermore an expensive technique since it can only be performed at large-scale facilities. In contrast, SAXS and SLS are cheaper techniques that can be available at the home laboratory, as they have been for the present study.

This work was supported by a grant from the Danish Natural Science Council.

\section{References}

Aharoni, S. M. (1983). Macromolecules, 16, 1722-1728.

Balsara, N. P., Tirrell, M. \& Lodge, T. P. (1991). Macromolecules, 24, 1975-1986.

Bracewell, R. N. (2000). The Fourier Transform and its Applications, pp. 219-224. Singapore: McGraw-Hill.

Cammas, S., Suzuki, K., Sone, C., Sakurai, Y., Kataoka, K. \& Okano, T. (1997). J. Controlled Release, 48, 157-164.

Cates, M. E. (1987). Macromolecules, 20, 2289-2296.

Cloizeaux, J. des \& Jannink, G. (1990). J. Phys. Condens. Matter, 2, 124.

Derici, L., Ledger, S., Mai, S.-M., Booth, C., Hamley, I. W. \& Pedersen, J. S. (1999). Phys. Chem. Chem. Phys. 1, 2773-2785.

Fetters, L. J., Lohse, D. J., Richter, D., Witten, T. A. \& Zirkel, A. (1994). Macromolecules, 27, 4639-4647.

Förster, S., Zisenis, M., Wenz, E. \& Antonietti, M. (1996). J. Chem. Phys. 104, 9956-9970.

Garamus, V. M., Pedersen, J. S., Kawasaki, H. \& Maeda, H. (2000). Langmuir, 16, 6431-6437.

Glatter, O. (1977). J. Appl. Cryst. 10, 415-421.

Glatter, O. \& Kratky, O. (1982). Editors. Small Angle X-ray Scattering, pp. 136-137. New York: Academic Press.

Hamley, I. W. (2005). Block Copolymers in Solution; Fundamentals and Applications. Chichester: Wiley.

Hanley, K. J., Lodge, T. P. \& Huang, C.-I. (2000). Macromolecules, 33, 5918-5931.

Hawker, C. J. \& Wooley, K. L. (2005). Science, 309, 1200-1205.

Kaya, H., Willner, L., Allgaier, J., Stellbrink, J. \& Richter, D. (2002). Appl. Phys. A, 74, S499-S501.

Kinning, D. J. \& Thomas, E. L. (1984). Macromolecules, 17, 17121718.

Laurati, M., Stellbrink, J., Lund, R., Willner, L., Zaccarelli, E. \& Richter, D. (2007). Phys. Rev. E, 76, 041503.

Lee, A. S., Bütün, V., Vamvakaki, M., Armes, S. P., Pople, J. A. \& Gast, A. P. (2002). Macromolecules, 35, 8540-8551.

Leibler, L., Orland, H. \& Wheeler, J. C. (1983). J. Chem. Phys. 79, 3550-3557.

Lodge, T. L., Bang, J., Li, Z., Hillmyer, M. A. \& Talmon, Y. (2005). Faraday Discuss. 128, 1-12.

Lund, R., Willner, L., Stellbrink, J., Radulescu, A. \& Richter, D. (2004). Macromolecules, 37, 9984-9993.

Moore, P. B. (1980). J. Appl. Cryst. 13, 168-175.

Müller, H., Leube, W., Tauer, K., Förster, S. \& Antonietti, M. (1997). Macromolecules, 30, 2288-2293.

Nagarajan, R. \& Ganesh, K. (1989). J. Chem. Phys. 90, 5843-5856.

Ndoni, S., Papadakis, C. M., Bates, F. S. \& Almdal, K. (1995). Rev. Sci. Instrum. 66, 1090-1095.

Neugebauer, T. (1943). Annal. Phys. 42, 509-533.

Ohta, T. \& Oono, Y. (1982). Phys. Lett. A, 89, 460-464.

Pedersen, J. S. (1993). J. Phys. (Paris) IV, 3, 491-498.

Pedersen, J. S. (1997). Adv. Colloid Interface Sci. 70, 171-210.

Pedersen, J. S. (2000). J. Appl. Cryst. 33, 637-640.

Pedersen, J. S. (2004). J. Appl. Cryst. 37, 369-380.

Pedersen, J. S. \& Gerstenberg, M. C. (1996). Macromolecules, 29, 1363-1365.

Pedersen, J. S., Hansen, S. \& Bauer, R. (1994). Eur. Biophys. J. 22, 379-389.

Pedersen, J. S., Laso, M. \& Schurtenberger, P. (1996). Phys. Rev. E, 54, R5917-R5920. 


\section{research papers}

Pedersen, J. S., Posselt, D. \& Mortensen, K. (1990). J. Appl. Cryst. 23, 321-333.

Pedersen, J. S. \& Schurtenberger, P. (1996a). J. Appl. Cryst. 29, 646661.

Pedersen, J. S. \& Schurtenberger, P. (1996b). Macromolecules, 29, 7602-7612.

Pedersen, J. S. \& Schurtenberger, P. (1999). Europhys. Lett. 45, 666672.

Pedersen, J. S., Svaneborg, C., Almdal, K., Hamley, I. W. \& Young, R. N. (2003). Macromolecules, 36, 416-433.

Poppe, A., Willner, L., Allgaier, J., Stellbrink, J. \& Richter, D. (1997). Macromolecules, 30, 7462-7471.

Porod, G. (1948). Acta Phys. Austriaca, 2, 255-292.

Pusey, P. N. (2002). Neutrons, X-rays and Light: Scattering Methods Applied to Soft Condensed Matter, edited by P. Lindner \& T. Zemb, pp. 16-18. Amsterdam: North-Holland, Elsevier.
Rayleigh (1910). Proc. R. Soc. London Ser. A, 84, 25-46.

Shannon, C. E. \& Weaver, W. (1959). The Mathematical Theory of Communication, p. 53. Urbana: University of Illinois Press.

Sommer, C., Pedersen, J. S. \& Stein, P. C. (2004). J. Phys. Chem. B, 108, 6242-6249.

Stellbrink, J., Rother, G., Laurati, M., Lund, R., Willner, L. \& Richter, D. (2004). J. Phys. Condens. Matter, 16, S3821-S3834.

Svaneborg, C. \& Pedersen, J. S. (2001). Phys. Rev. E, 64, 010802.

Svaneborg, C. \& Pedersen, J. S. (2002). Macromolecules, 35, 10281037.

Wang, S.-C., Wang, C.-K., Chang, F.-M. \& Tsao, H.-K. (2002). Macromolecules, 35, 9551-9555.

Willner, L., Poppe, A., Allgaier, J., Monkenbusch, M., Lindner, P. \& Richter, D. (2000). Europhys. Lett. 51, 628-634.

Zhulina, E. B., Adam, M., LaRue, I., Sheiko, S. S. \& Rubinstein, M. (2005). Macromolecules, 38, 5330-5351. 